\title{
Respuesta fisiológica y de crecimiento en plantas de Quillaja saponaria y Cryptocarya alba sometidas a restricción hídrica
}

\author{
Physiological and growth response in Quillaja saponaria and Cryptocarya alba plants \\ under restricted water conditions
}

\author{
Sergio Donoso ${ }^{\text {a* }}$, Karen Peña a, Cristian Pacheco a, Gabriela Luna a , Aldo Aguirre ${ }^{a}$ \\ * Autor de correspondencia: a Universidad de Chile, Facultad de Ciencias Forestales y Conservación de la Naturaleza, \\ Departamento de Silvicultura, CC 9206, Santiago, Chile, tel.: (56-2) 9785918, fax: (56-2) 5417955, sedonoso@uchile.cl
}

\begin{abstract}
SUMMARY
Central Chile is characterized by a Mediterranean Climate. Some of the tree species that grows in this zone (e.g. Quillaja saponaria (quillay) and Cryptocarya alba (peumo)) have developed physiological mechanisms to tolerate drought periods during the summer. The aim of this study was to quantify physiological and growth parameters in two-year-old quillay and peumo saplings, maintained under controlled water restricted conditions during one summer season. The plants were assigned to two different experimental treatments: well watered (control-WW) and restricted irrigation (RI). Saplings in RI had an average predawn water potential $\left(\psi_{\text {pd }}\right)$ near to -3.5 MPa. At the end of the water restriction period, plant gas exchange parameters were determined and biomass distributions in leaves, branches, stems and roots were analyzed. During the period of high water deficit, quillay did not showed a clear mechanism of adjustment to water deficit, but a reduction in $55 \%$ of leaf biomass decreased the evapotranspiration rate. Peumo showed an osmotic adjustment. Net photosynthesis, stomatal conductance and transpiration rate of quillay and peumo plants were significantly reduced in the RI treatment when compared to control saplings. Quillay saplings showed a significant reduction in the above/below biomass ratio under the restricted irrigation treatment (2.3 to 1.4). In contrast peumo saplings did not change significantly this ratio (1.3).
\end{abstract}

Key words: Quillaja saponaria, Cryptocarya alba, water potential, gas exchange, biomass distribution.

\section{RESUMEN}

La zona central de Chile presenta un clima mediterráneo donde las especies arbóreas como Quillaja saponaria (quillay) y Cryptocarya alba (peumo) han desarrollado mecanismos fisiológicos que le permiten tolerar la falta de agua durante el verano. Se desarrolló un ensayo con plantas de dos años de quillay y peumo, que fueron sometidas a condiciones de restricción hídrica, en el periodo estival. Un grupo de plantas fue regado diariamente a capacidad de campo (tratamiento testigo) y otro grupo fue sometido a riego restringido hasta llegar a un potencial hídrico a pre-alba cercano a -3,5 MPa. Finalizado el período de restricción hídrica, las plantas se rehidrataron. Durante el estudio se midieron variables hídricas y de crecimiento. Al finalizar el periodo de restricción hídrica se determinaron tasas de intercambio gaseoso, así como la distribución de biomasa en hojas, ramas, fuste y raíces. En el periodo de mayor restricción hídrica, quillay no presentó un mecanismo claro de ajuste al déficit hídrico, pero redujo su biomasa foliar en $55 \%$ para reducir la pérdida hídrica. Mientras que peumo presentó ajuste osmótico. Quillay y peumo presentaron una reducción significativa de la fotosíntesis neta, conductancia estomática y transpiración de frente a riego restringido. La relación parte aérea/parte radical no se modificó en peumo $(1,3)$, y se redujo significativamente en quillay, de 2,3 a 1,4 para el tratamiento de restricción hídrica.

Palabras clave: Quillaja saponaria, Cryptocarya alba, potencial hídrico, intercambio gaseoso, distribución de biomasa.

\section{INTRODUCCIÓN}

Dos de las especies siempre verdes más importantes de la zona mediterránea chilena son quillay (Quillaja saponaria Mol.) y peumo (Cryptocarya alba Mol. Looser). Ambas se desarrollan entre las regiones de Coquimbo $\left(30^{\circ}\right.$ latitud S) y de La Araucanía (38 latitud S) de Chile, y es común encontrarlas desarrollándose de forma conjunta en algunas áreas de su distribución. Quillay tolera suelos pobres, altas fluctuaciones térmicas y condiciones extremas de sequía. Peumo, en tanto, se desarrolla preferentemente asociado a quebradas y sitios húmedos, y en laderas de exposición sur en su distribución más septentrional, aunque es posible hallarlo en terrenos con fuerte insolación pero con cierto grado de humedad (Rodríguez et al. 1983).

Los ecosistemas con clima mediterráneo se caracterizan por presentar diversos tipos de estrés que restringen el crecimiento y sobrevivencia de las plantas (Cabrera 2002). En particular, la zona mediterránea de Chile se caracteriza por presentar una marcada estacionalidad en el monto de precipitaciones, concentradas en los meses de invierno. Durante el periodo estival, la baja disponibilidad hídrica del suelo, en conjunto con las altas temperaturas y radiación solar de la estación, favorecen la aparición de múltiples estreses en las plantas.

El estrés hídrico es uno de los factores más limitantes 
del crecimiento, la composición y distribución de las especies que se desarrollan en climas mediterráneos (Chaves et al. 2002, Otieno et al. 2005). El déficit hídrico afecta varios aspectos del funcionamiento de las plantas, desde el metabolismo celular (incluyendo la fotosíntesis) hasta el crecimiento. La fotosíntesis es el proceso fisiológico de mayor envergadura en el desempeño de las plantas. La tasa de fotosíntesis, tanto de las plantas C3 como de las C4, decrece a medida que su contenido hídrico relativo y el potencial hídrico disminuyen (Lawlor 2002). En este sentido, Cabrera (2002) menciona que tanto quillay como litre (Lithraea caustica Molina Hook. et Arn.) mantienen un balance de carbono positivo aún bajo restricción hídrica. Este comportamiento contrasta con peumo, que presenta un balance negativo debido a que la fotosíntesis es muy baja con restricción hídrica (Martínez y Armesto 1983). Por otra parte, el cierre estomático es el primer paso en la respuesta ante la restricción hídrica. Una menor conductancia estomática reduce la pérdida de agua, disminuye la tasa de desarrollo del estrés y minimiza su severidad (Raftoyannis y Radoglou 2002), pero reduce la absorción de $\mathrm{CO}_{2}$. Todas estas respuestas tienen como consecuencia la disminución del crecimiento global de la planta y, por ende, una disminución en la acumulación de biomasa.

El transporte de agua en la planta es una parte integral del proceso de crecimiento, ya que una variedad de procesos relacionados con el crecimiento, incluyendo la formación y expansión de células vegetales, son dependientes de la presión de turgencia y el volumen celular (Woodruff et al. 2004). Por tanto, las relaciones existentes entre las variables hídricas, fotosíntesis y conductancia estomática son aspectos clave en la comprensión del proceso de tolerancia al estrés (Dickson y Tomlinson 1996), lo cual determina a su vez el crecimiento, reproducción, distribución y composición de especies vegetales.

El estudio de estas relaciones además, es de importancia para el manejo de plantaciones forestales, pues provee información básica para la toma de decisiones. Gebrekirstos et al. (2006) mencionan que la falta de conocimiento en la elección de especies para determinados ambientes lleva al fracaso de los programas de reforestación, por lo que se ha enfatizado la necesidad de investigar dichas relaciones y sus respuestas ante cambios en la disponibilidad del recurso.

En bosques esclerófilos, el desarrollo conjunto de quillay y peumo se atribuye a que presentan diferentes mecanismos de respuesta frente a periodos de estrés hídrico. El objetivo de este estudio fue evaluar las respuestas morfofisiológicas presentadas por quillay y peumo bajo diferentes condiciones de restricción hídrica controlada, mediante el análisis de variables hídricas, de intercambio gaseoso, crecimiento y de distribución de biomasa.

\section{MÉTODOS}

Se realizó un ensayo en condiciones de restricción hídrica controlada en un vivero ubicado en la región Metropolitana de la zona central de Chile ( $33^{\circ}$ latitud S), con 40 individuos seleccionados por especie (quillay y peumo), de dos años de edad, los cuales fueron traspasados a macetas de 9 litros de capacidad y llenadas con una mezcla de suelo del lugar, de textura franco arenosa (40\%), perlita (30\%), arena (30\%) y fertilizante. De los 40 individuos de cada especie se seleccionaron aleatoriamente 15 que fueron asignados a un tratamiento testigo con aporte hídrico regular, y los restantes fueron sometidos a restricción hídrica controlada, como se describe a continuación.

El seguimiento y control de la pérdida de humedad del sustrato se hizo cada dos días mediante la determinación del contenido hídrico del sustrato en la maceta (CHS) [ecuación 1], mediante mediciones de su peso con una balanza cuya precisión era de 0,1 kg.

$$
C H S=\frac{P F-P S}{P H-P S} \times 100
$$

Donde,

CHS = contenido hídrico del sustrato en la maceta (\%).

$P F=$ Peso fresco de la maceta al momento de la medición (kg).

$P H=$ Peso hidratado de la maceta (kg), obtenido después de ser regada y drenada el agua gravitacional.

$P S=$ Peso seco de la maceta $(\mathrm{kg})$.

Las plantas del tratamiento testigo se mantuvieron con valores de contenido hídrico del sustrato entre 75 y 100 $\%$ durante todo el ensayo, mientras que a los individuos del tratamiento de restricción se les suspendió el riego y fueron evaluados periódicamente a medida que el contenido hídrico del sustrato disminuía paulatinamente hasta llegar aproximadamente a un valor del $40 \%$ (con respecto al peso inicial al suspender el riego). Luego, la suspensión del riego se prolongó durante un mes en ambas especies (mantención de restricción hídrica), hasta alcanzar un potencial hídrico de pre-alba cercano a -3,5 $\mathrm{MPa}$, este valor se basó en estudios hechos en especies mediterráneas (Vilagrosa et al. 2005 y Gallé et al. 2007). Una vez transcurrido el periodo total de restricción hídrica, y con el objeto de observar su capacidad de recuperación, las plantas del tratamiento de restricción fueron regadas a saturación y mantenidas bajo esta condición durante aproximadamente un mes (rehidratación).

Para caracterizar y comparar la condición hídrica se utilizaron seis individuos por especie y periodo de medición, a los que se les midió el potencial hídrico foliar $\left(\Psi_{\mathrm{a}}\right)$ y el contenido hídrico relativo foliar a pre-alba $\left(\mathrm{CHR}_{\mathrm{a}}\right)$ (Lawlor y Cornic 2002). El potencial hídrico foliar fue determinado con una bomba de presión PMS Instrument modelo 1000, mientras que el contenido hídrico relativo foliar se calculó con los pesos fresco (PF), hidratado (PH) 
y seco (PS) de las muestras. Las mediciones de potencial hídrico foliar y contenido hídrico relativo foliar a pre-alba se hicieron al inicio del ensayo y al final de los periodos de mantención de la restricción hídrica y rehidratación, en ramillas ubicadas en el tercio superior de cada planta, procurando que fueran similares en número, madurez y tamaño de hojas.

Con parte del material colectado para medir potencial hídrico foliar y contenido hídrico relativo foliar a pre-alba (cuatro ramillas por especie, por tratamiento y periodo de medición), y con el objeto de conocer otros parámetros relacionados con el comportamiento hídrico de las plantas, se construyeron curvas de presión-volumen siguiendo los procedimientos descritos por Lenz et al. (2006). Estos otros parámetros fueron: potencial osmótico a cero turgencia $\left(\pi_{0}\right)$ y a turgencia máxima $\left(\pi_{100}\right)$, módulo de elasticidad de la pared celular $(\varepsilon)$ y contenido hídrico relativo a cero turgencia $\left(\mathrm{CHR}_{0}\right)$.

Antes de iniciar el periodo de rehidratación, en cinco individuos por tratamiento y por especie, se determinaron alrededor del mediodía (PAR superior a $1300 \mu \mathrm{mol} \mathrm{m}^{-2} \mathrm{~s}^{-1}$ ) las siguientes variables de intercambio gaseoso: tasa de fotosíntesis neta $\left(\mathrm{A}_{\mathrm{n}}\right)$, conductancia estomática $\left(\mathrm{g}_{\mathrm{s}}\right)$, tasa de transpiración (E) y concentración interna de $\mathrm{CO}_{2}\left(\mathrm{C}_{\mathrm{i}}\right)$. Estas mediciones se efectuaron con un aparato portátil de fotosíntesis (ADC-LCi, UK).

El efecto de los tratamientos aplicados en el crecimiento se determinó mediante el diámetro a la altura del cuello (DAC) y longitud (L) de la rama del eje principal de cada planta, en plantas que fueron previamente marcadas al inicio del ensayo. Estas mediciones se hicieron con un pie de metro con precisión de 0,1 mm y una cinta métrica con precisión de 0,1 cm, respectivamente. La oportunidad de estas mediciones coincidió con las mediciones de potencial hídrico foliary contenido hídrico relativo foliar a pre-alba.

Al término del periodo de mantención de la restricción hídrica, se pesó la biomasa aérea y radical de tres individuos de cada tratamiento y de cada especie. Cada planta muestreada se separó en sistema radical y parte aérea, y ésta a su vez se dividió en hojas y material leñoso. Estas muestras secadas a $65^{\circ} \mathrm{C}$ en una estufa de aire forzado hasta alcanzar un peso constante. Con estos datos se calculó la biomasa total y por componente, y se compararon con la biomasa registrada al inicio del ensayo de tres plantas por especie.

El ensayo fue conducido de acuerdo con un diseño completamente aleatorio. Las distintas variables obtenidas fueron sometidos a análisis de varianza (ANDEVA) con un nivel $P=0,05$, y la medias se compararon mediante la prueba de rango múltiple de Duncan, cuando hubo diferencias estadísticas. También se calculó para cada especie el coeficiente de correlación ( $\mathrm{r}$ de Pearson) entre potencial hídrico a pre-alba y el contenido hídrico del sustrato. Para el procesamiento de los datos se utilizó el programa estadístico SPSS 11.5 para Windows.

\section{RESULTADOS}

Relaciones hídricas. El contenido hídrico del sustrato en las macetas del tratamiento testigo en quillay presentó,

Cuadro 1. Parámetros hídricos de plantas de Quillaja saponaria (quillay) y Cryptocarya alba (peumo) según periodo de evaluación y tratamiento. Contenido hídrico del sustrato (CHS); potencial hídrico en hojas a pre-alba ( $\left.\Psi_{\mathrm{a}}\right)$; contenido hídrico relativo a pre-alba $\left(\mathrm{CHR}_{\mathrm{a}}\right)$. Valores corresponden a medias \pm el error estándar $(\mathrm{n}=6)$. Letras distintas indican diferencias significativas entre los tratamientos $(P<0,05)$.

Soil water contents (CHS); predawn leaf water potential $\left(\mathrm{Y}_{\mathrm{pD}}\right)$ and predawn relative water content $\left(\mathrm{CHR}_{\mathrm{a}}\right)$ of quillay and peumo plants as a function of evaluation period and treatment. Values are mean $\pm \mathrm{SE}$ of six measurements per species and treatment. Significant differences $(P<0.05)$ are indicated by different letters.

\begin{tabular}{|c|c|c|c|c|c|}
\hline Especie & Periodo & Tratamientos & CHS (\%) & $\Psi_{\mathrm{a}}(\mathrm{Mpa})$ & $\mathrm{CHR}_{\mathrm{a}}(\%)$ \\
\hline \multirow{6}{*}{ Quillay } & \multirow{2}{*}{$\begin{array}{l}\text { Inicio de la restricción } \\
\text { hídrica }\end{array}$} & Testigo & $81 \pm 4,6$ & $-0,7 \pm 0,1 \mathrm{a}$ & $94,5 \pm 1,8 \mathrm{a}$ \\
\hline & & Restricción hídrica & $40 \pm 3,6$ & $-1,3 \pm 0,1 b$ & $91,3 \pm 0,9 b$ \\
\hline & \multirow{2}{*}{$\begin{array}{l}\text { Mantención de la res- } \\
\text { tricción hídrica }\end{array}$} & Testigo & $82 \pm 2,5$ & $-0,5 \pm 0,0 \mathrm{a}$ & $93,0 \pm 0,5 \mathrm{a}$ \\
\hline & & Restricción hídrica & $40 \pm 3,1$ & $-3,9 \pm 0,5 b$ & $83,2 \pm 2,9 b$ \\
\hline & \multirow{2}{*}{ Rehidratación } & Testigo & $93 \pm 3,9$ & $-0,5 \pm 0,1 \mathrm{a}$ & $83,8 \pm 5,6 b$ \\
\hline & & Restricción hídrica & $97 \pm 1,4$ & $-0,5 \pm 0,1 \mathrm{a}$ & $97,5 \pm 1,0 \mathrm{a}$ \\
\hline \multirow{6}{*}{ Peumo } & \multirow{2}{*}{$\begin{array}{l}\text { Inicio de la restricción } \\
\text { hídrica }\end{array}$} & Testigo & $100 \pm 0,0$ & $-0,6 \pm 0,0 \mathrm{a}$ & $99,0 \pm 1,0 \mathrm{a}$ \\
\hline & & Restricción hídrica & $45 \pm 4,7$ & $-2,7 \pm 0,3 b$ & $90,0 \pm 3,0 \mathrm{~b}$ \\
\hline & \multirow{2}{*}{$\begin{array}{l}\text { Mantención de la res- } \\
\text { tricción hídrica }\end{array}$} & Testigo & $99 \pm 1,3$ & $-0,4 \pm 0,1 \mathrm{a}$ & $100,0 \pm 1,0 \mathrm{a}$ \\
\hline & & Restricción hídrica & $43 \pm 4,3$ & $-3,6 \pm 0,5 b$ & $97,0 \pm 2,0 \mathrm{a}$ \\
\hline & \multirow{2}{*}{ Rehidratación } & Testigo & $74 \pm 2,1$ & $-0,7 \pm 0,1 \mathrm{a}$ & $99,0 \pm 1,0 \mathrm{a}$ \\
\hline & & Restricción hídrica & $91 \pm 3,0$ & $-0,6 \pm 0,1 \mathrm{a}$ & $100,0 \pm 2,0 \mathrm{a}$ \\
\hline
\end{tabular}


durante todo el ensayo, valores de 81 a $93 \%$, cercanos a $100 \%$, mientras que en peumo fluctuaron entre $100 \mathrm{y}$ $74 \%$ (cuadro 1). Dado el riego permanente que recibieron las plantas testigo, el potencial hídrico foliar pre-alba alcanzó valores en torno a -0,5 MPa para ambas especies, mientras que el contenido hídrico relativo foliar a pre-alba fue de $90 \%$ en promedio en quillay y de $99 \%$ en peumo. Por su parte, el tratamiento de restricción hídrica controlada mostró, durante el periodo de inicio de restricción hídrica, una disminución progresiva del contenido hídrico del sustrato hasta alcanzar valores de $45 \%$ aproximadamente en ambas especies, luego de dos semanas. La disminución de esta variable estuvo acompañada por la disminución del potencial hídrico foliar a pre-alba y del contenido hídrico relativo foliar a pre-alba, que en el caso de quillay llegó a valores de $-1,3 \mathrm{MPa}$ y $91 \%$, respectivamente, mientras que en peumo se alcanzaron valores de $-2,7 \mathrm{MPa}$ y $90 \%$, respectivamente. Durante el periodo de mantenimiento de la restricción hídrica, el contenido hídrico del sustrato en el tratamiento de restricción hídrica se mantuvo en torno a $40 \%$ en ambas especies. A medida que la restricción se intensificó, los valores de potencial hídrico foliar a prealba se redujeron más (-3,9 MPa en quillay y -3,6 MPa en peumo), con diferencias significativas $(P<0,05)$ respecto al testigo (cuadro 1). Durante el periodo de rehidratación, el potencial hídrico foliar a pre-alba volvió a los valores observados al inicio del ensayo, sin diferencias significativas entre tratamientos. Los coeficientes de correlación entre el potencial hídrico a pre-alba y el contenido hídrico del sustrato fueron de $0,78(P<0,01)$ en quillay y 0,86 en peumo $(P<0,01)$.

Parámetros hídricos derivados de las curvas presión-volumen. En quillay, los potenciales osmóticos a cero turgencia $\left(\pi_{0}\right)$ y a turgencia máxima $\left(\pi_{100}\right)$ mostraron diferencias que no fueron significativas entre tratamientos durante los tres periodos de evaluación. Sin embargo, hubo diferencias significativas dentro del tratamiento, con valores más bajos en el periodo de inicio de la restricción hídrica que en el periodo de recuperación del estrés hídrico (cuadro 2 ). En peumo, el $\pi_{0}$ durante el periodo de restricción hídrica fue significativamente menor en el tratamiento de restricción hídrica (-4,3 MPa) que en el tratamiento testigo (-3,5 MPa). En ambas especies no se detectaron diferencias entre tratamientos en el módulo de elasticidad de la pared celular $(\varepsilon)$ durante el periodo de restricción hídrico, pero sí en el periodo de rehidratación, donde las plantas de quillay del tratamiento con restricción hídrica mantuvieron la rigidez de sus paredes durante todo el ensayo, no así en las plantas bien regadas las que al final del período de rehidratación disminuyeron su módulo de elasticidad. Mientras que las paredes celulares de peumo se tornaron más elásticas en las plantas sometidas a estrés hídrico. Considerando el contenido hídrico relativo a cero turgencia, éste aumentó ante el estrés hídrico en quillay indicando una menor capacidad de mantenimiento de la turgencia en esta situa-

Cuadro 2. Parámetros hídricos derivados de las curvas presión-volumen. Valores medios \pm error estándar de las plantas de Quillaja saponaria (quillay) y Cryptocarya alba (peumo), según periodo de evaluación y tratamiento $(\mathrm{n}=4)$. Potencial osmótico a pleno turgencia $\left(\pi_{100}\right)$; potencial osmótico a cero turgencia $\left(\pi_{0}\right)$; contenido hídrico relativo a cero turgencia $\left(\mathrm{CHR}_{0}\right)$; módulo de elasticidad $(\varepsilon)$. Letras mayúsculas distintas indican diferencias significativas entre tratamientos, y letras minúsculas distintas indican diferencias significativas dentro del tratamiento $(P<0,05)$.

Water relation parameters derived from the pressure-volume curves. Osmotic potential at full turgor $\left(\mathrm{p}_{100}\right)$; osmotic potential at turgor loss point $\left(\mathrm{p}_{0}\right)$; relative water content at turgor loss point $\left(\mathrm{CHR}_{0}\right)$ and bulk elastic modulus $(\varepsilon)$ per species, treatment and evaluation period. Values are mean \pm SE of six measurements per species and treatment. Different capital letters indicate significant differences between treatments and different lower case letters indicate significant differences between evaluation periods $(P<0.05)$.

\begin{tabular}{cllcccc}
\hline \multirow{2}{*}{ Especie } & \multicolumn{1}{c}{ Periodo } & \multicolumn{1}{c}{ Tratamiento } & $\pi_{100}(\mathrm{Mpa})$ & $\pi_{0}(\mathrm{Mpa})$ & $\mathrm{CHR}_{0}(\%)$ & $\mathrm{E}$ \\
\hline \multirow{2}{*}{ Quillay } & Inicio de la res- & Testigo & $-3,0 \pm 0,1 \mathrm{Aa}$ & $-3,5 \pm 0,1 \mathrm{Aa}$ & $81,8 \pm 1,6 \mathrm{Aa}$ & $15,2 \pm 1,9 \mathrm{Aa}$ \\
& tricción hídrica & Restricción hídrica & $-3,0 \pm 0,3 \mathrm{Aa}$ & $-3,5 \pm 0,4 \mathrm{Aa}$ & $83,8 \pm 7,2 \mathrm{Aa}$ & $17,6 \pm 1,9 \mathrm{Aa}$ \\
\cline { 2 - 7 } & Mantención de la & Testigo & $-2,7 \pm 0,1 \mathrm{Aa}$ & $-3,4 \pm 0,0 \mathrm{Aa}$ & $81,9 \pm 1,8 \mathrm{Aa}$ & $14,1 \pm 1,5 \mathrm{Aa}$ \\
& restricción hídrica & Restricción hídrica & $-2,6 \pm 0,2 \mathrm{Aab}$ & $-3,2 \pm 0,2 \mathrm{Aab}$ & $88,6 \pm 1,8 \mathrm{Ba}$ & $17,7 \pm 2,2 \mathrm{Aa}$ \\
\cline { 2 - 7 } & Rehidratación & Testigo & $-1,8 \pm 0,2 \mathrm{Ab}$ & $-2,6 \pm 0,3 \mathrm{Ab}$ & $74,7 \pm 7,0 \mathrm{Ab}$ & $7,6 \pm 2,1 \mathrm{Ab}$ \\
& & Restricción hídrica & $-2,1 \pm 0,2 \mathrm{Ab}$ & $-2,6 \pm 0,3 \mathrm{Ab}$ & $89,5 \pm 1,4 \mathrm{Ba}$ & $16,1 \pm 1,1 \mathrm{Ba}$ \\
\hline \multirow{2}{*}{ Peumo } & Inicio de la res- & Testigo & $-3,4 \pm 0,2 \mathrm{Aa}$ & $-3,6 \pm 0,3 \mathrm{Aa}$ & $88,0 \pm 2,0 \mathrm{Aa}$ & $22,8 \pm 2,2 \mathrm{Aa}$ \\
& tricción hídrica & Restricción hídrica & $-3,0 \pm 0,1 \mathrm{Aa}$ & $-3,6 \pm 0,1 \mathrm{Aa}$ & $85,0 \pm 2,0 \mathrm{Aa}$ & $20,9 \pm 2,9 \mathrm{Aab}$ \\
\cline { 2 - 7 } & Mantención de la & Testigo & $-3,3 \pm 0,2 \mathrm{Aa}$ & $-3,5 \pm 0,1 \mathrm{Aa}$ & $88,0 \pm 2,0 \mathrm{Aa}$ & $27,9 \pm 2,7 \mathrm{Aa}$ \\
& restricción hídrica & Restricción hídrica & $-3,4 \pm 0,2 \mathrm{Aa}$ & $-4,3 \pm 0,2 \mathrm{Bb}$ & $87,0 \pm 1,0 \mathrm{Aa}$ & $23,4 \pm 1,9 \mathrm{Aa}$ \\
\cline { 2 - 7 } & Rehidratación & Testigo & $-2,9 \pm 0,2 \mathrm{Aa}$ & $-3,4 \pm 0,2 \mathrm{Aa}$ & $90,0 \pm 2,0 \mathrm{Aa}$ & $23,6 \pm 1,2 \mathrm{Aa}$ \\
& & Restricción hídrica & $-3,2 \pm 0,1 \mathrm{Aa}$ & $-3,9 \pm 0,1 \mathrm{Aa}$ & $83,0 \pm 2,0 \mathrm{Ba}$ & $14,1 \pm 1,5 \mathrm{Bb}$ \\
\hline
\end{tabular}


ción durante todo el ensayo. Por el contrario, en peumo, se mantuvo el contenido hídrico relativo a cero turgencia durante el periodo de mantención de la restricción hídrica y luego de la rehidratación disminuyó, posiblemente como producto de la mayor elasticidad de la pared celular, esto también se observa en las plantas de quillay bien regadas al término del periodo de rehidratación.

Intercambio gaseoso. Las plantas del tratamiento testigo de ambas especies presentaron valores de intercambio gaseoso significativamente diferentes a las que se desarrollaron bajo condiciones de riego restringido (figura 1). En quillay los valores disminuyeron $84,5 \%, 98,4 \%, 94,1 \%$ y $58,9 \%$ en fotosíntesis neta, conductancia estomática, transpiración y concentración interna de $\mathrm{CO}_{2}$, respectivamente. En peumo los valores de fotosíntesis neta, conductancia estomática y transpiración fueron $94,4 \%, 99,8 \%$, $100 \%$ menores en el tratamiento de restricción hídrica que en el tratamiento testigo, respectivamente, mientras que la concentración interna de $\mathrm{CO}_{2}$ fue un 104,4 \% mayor en el tratamiento de restricción hídrica que en el tratamiento testigo.

Crecimiento. En las plantas del tratamiento testigo el diámetro a la altura del cuello se incrementó durante todo el ensayo, tanto en quillay como en peumo. Por el contrario, en las plantas sometidas a estrés hídrico el engrosamiento del tallo fue significativamente inferior al del tratamiento testigo (figuras $2 \mathrm{~A}$ y $2 \mathrm{~B}$ ). En el tratamiento testigo el incremento en diámetro de cuello registrado entre el inicio y el término del ensayo, fue de $2,2 \mathrm{~mm}$ en quillay y $1,7 \mathrm{~mm}$ en peumo, mientras que las plantas del tratamiento de restricción hídrica experimentaron un decrecimiento de $0,1 \mathrm{~mm}$ en quillay y un incremento casi nulo en el caso de peumo. Un patrón similar se observó en el crecimiento en longitud, en ambas especies (figuras $2 \mathrm{C}$ y $2 \mathrm{D}$ ). $\mathrm{Al}$ comparar el crecimiento en longitud entre el inicio y término del ensayo, las plantas del tratamiento testigo en quillay crecieron $12 \mathrm{~cm}$ y en peumo este valor fue de $8 \mathrm{~cm}$. En tanto, las plantas del tratamiento de restricción hídrica de quillay sólo crecieron 2,6 cm y en peumo la muerte de tejidos juveniles en los ápices principales determinó la disminución de la longitud total de las plantas en $0,3 \mathrm{~cm}$.

Distribución de la biomasa. En ambas especies, tanto la biomasa foliar como la leñosa aérea (tallo y ramas) experimentaron un incremento significativo en las plantas del tratamiento testigo, respecto a la condición inicial; en cambio, las plantas del tratamiento de restricción hídrica no mostraron diferencias significativas en el mismo periodo (cuadro 3). En quillay, la proporción de biomasa foliar respecto de la total en el tratamiento de restricción hídrica, disminuyó desde $37 \%$ al inicio del ensayo hasta $16 \%$ al final del ensayo; en peumo, los valores fueron $25 \%$ y $15 \%$, respectivamente. Sólo se observó una tendencia al aumento de la biomasa radical en el tratamiento de restric-
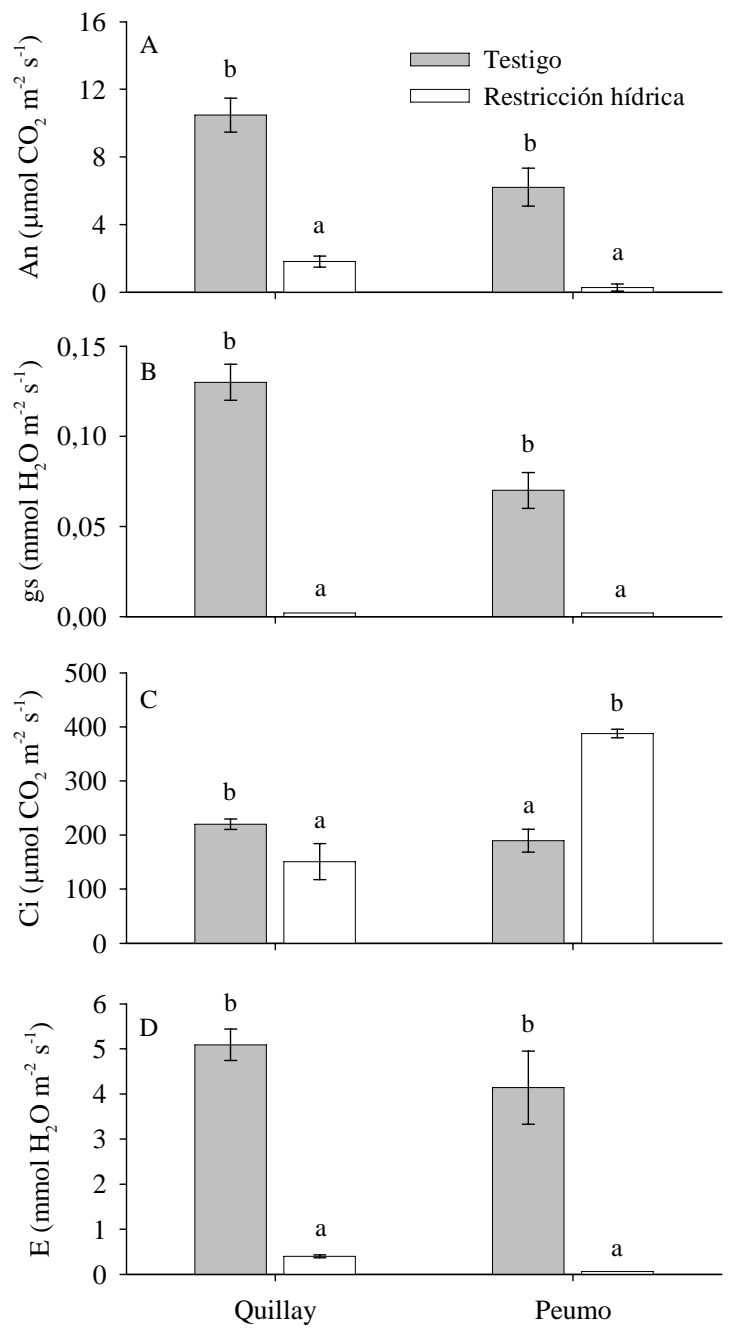

Figura 1. Tasas de intercambio gaseoso en plantas de Quillaja saponaria (quillay) y Cryptocarya alba (peumo) al finalizar el periodo de mantención de restricción hídrica. A) Fotosíntesis neta (An); B) conductancia estomática (gs); C) concentración interna de $\mathrm{CO}_{2}(\mathrm{Ci})$; D) transpiración (E). Valores corresponden a medias \pm el error estándar $(n=5)$. Letras distintas indican diferencias significativas entre tratamientos $(P<0,05)$.

Gas exchange rates in quillay and peumo plants at the end of the water restriction period. A) Net photosynthesis $\left(A_{n}\right)$; B) Stomatal conductance $\left(g_{s}\right)$; C) Intercellular $\mathrm{CO}_{2}$ concentration $\left(C_{i}\right)$; D) Transpiration $(E)$. Values are mean $\pm \mathrm{SE}$ of five measurements per species and treatment. Significant differences $(P<0.05)$ are indicated by different letters.

ción hídrica de quillay. Pese a ello, al término del ensayo la relación biomasa aérea/biomasa radical mostró diferencias significativas con respecto a la observada al inicio del ensayo. En peumo, la biomasa radical en el tratamiento de restricción hídrica casi no sufrió cambios, lo cual determinó que no se observara cambio en la relación biomasa aérea/biomasa radical. 

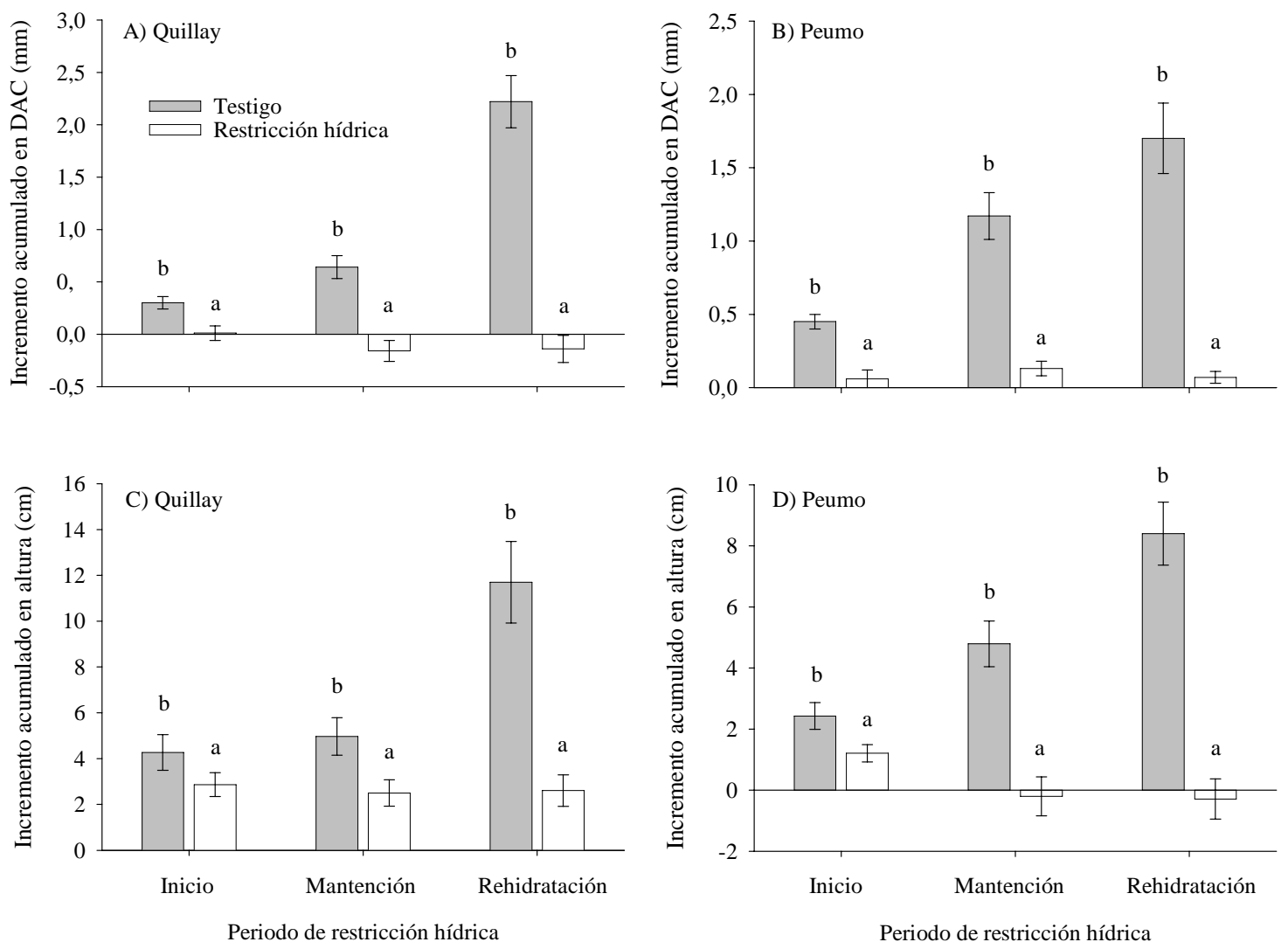

Figura 2. Crecimiento medio en diámetro a la altura del cuello (DAC) y altura de planta, por tratamiento de riego durante el ensayo, en Quillaja saponaria (quillay) (A y C) y Cryptocarya alba (peumo) (B y D). Valores corresponden a medias \pm el error estándar (n = 15-21). Letras distintas indican diferencias significativas entre tratamientos $(P<0,05)$.

Collar diameter increment (DAC) A) Quillay and B) Peumo, and height increment C) Quillay and D) Peumo per treatment during essay. Values are mean \pm SE of 15-21 measurements per species and treatment. Significant differences $(P<0.05)$ are indicated by different letters.

\section{DISCUSIÓN}

El comportamiento observado en el potencial hídrico a pre-alba y contenido hídrico relativo a pre-alba, tanto en plantas del tratamiento testigo como del tratamiento con riego restringido, concuerda con lo obtenido por diversos estudios en especies mediterráneas (Peña-Rojas et al. 2004). Estos resultados confirman que la disminución del potencial hídrico a pre-alba está relacionada fuertemente con la disminución del contenido hídrico del sustrato (correlaciones significativas en ambas especies), mientras que las variaciones del contenido hídrico relativo a prealba podrían estar más relacionadas con los mecanismos de regulación de la pérdida de agua. En cuanto a los mecanismos de ajuste osmótico y elástico de estas especies, las modificaciones de los parámetros derivados de las curvas presión-volumen observadas en los periodos de mantención de la restricción hídrica y de rehidratación, indican que peumo realiza ajuste osmótico, mientras que quillay, los valores menos negativos observado durante los periodos de restricción hídrica y de rehidratación en el potencial osmótico a cero turgencia y a turgencia máxima, al comparar entre los tratamientos y entre los periodos de estrés, indicaría que la especie no presenta la capacidad de realizar ajuste osmótico o elástico, y al encontrarse bajo condiciones de estrés hídrico severo, se produce la abscisión de sus hojas (cuadro 3), para disminuir la pérdida de agua y de este modo equilibrar su biomasa foliar con la eficiencia del sistema radical de suministrar agua a las hojas.

La senescencia inducida por el estrés hídrico de verano puede ser una adaptación de escape a un estrés mayor, lo cual indicaría una estrategia de tolerancia a sequía y este proceso juega un papel fundamental en la sobrevivencia de muchas especies, al contribuir a la mantención de un balance hídrico favorable en la planta (Munné-Bosch y Alegre 2004). En peumo, en tanto, las diferencias observadas entre los tratamientos en $\pi_{0}$ durante el periodo de mantención de la restricción hídrica, indican la presencia de ajuste osmótico, pues los valores más negativos observados en el tratamiento de restricción hídrica, son indicadores de una mayor concentración de solutos y con ello, se contribuiría al mantenimiento de la turgencia celular a 
Cuadro 3. Distribución de la biomasa en Quillaja saponaria (quillay) y Cryptocarya alba (peumo) según periodo de evaluación y tratamiento. Entre paréntesis el porcentaje respecto del total de biomasa. Letras mayúsculas distintas indican diferencias significativas entre tratamientos, y letras minúsculas distintas indican diferencias significativas entre inicio y final del ensayo $(P<0,05)$.

Biomass distribution of quillay and peumo plants as a function of evaluation period and treatments. Data in parenthesis correspond to the percentage of total dry matter. Different capital letters indicate significant differences between treatments and different lower case letters indicate significant differences within treatments $(P<0.05)$.

\begin{tabular}{|c|c|c|c|c|c|c|c|}
\hline \multirow[t]{2}{*}{ Especie } & \multirow{2}{*}{$\begin{array}{c}\text { Componente de } \\
\text { biomasa* }\end{array}$} & \multirow[t]{2}{*}{ Inicio del ensayo } & \multicolumn{5}{|c|}{ Final del ensayo } \\
\hline & & & \multicolumn{2}{|c|}{ Tratamiento testigo } & \multicolumn{2}{|c|}{ Tratamiento de restricción hídrica } & \\
\hline \multirow{5}{*}{ Quillay } & Hojas (g) & $16,9 \pm 1,0 \mathrm{a}$ & $(37 \%)$ & $43,0 \pm 7,3 \mathrm{bB}$ & $(33 \%)$ & $7,5 \pm 2,2 \mathrm{aA}$ & $(16 \%)$ \\
\hline & Ramas (g) & $14,9 \pm 1,5$ a & $(33 \%)$ & $42,7 \pm 4,5 \mathrm{bB}$ & $(33 \%)$ & $19,5 \pm 2,5 \mathrm{aA}$ & $(41 \%)$ \\
\hline & Raíz (g) & $13,9 \pm 0,7 \mathrm{a}$ & $(30 \%)$ & $43,5 \pm 3,4 \mathrm{bB}$ & $(34 \%)$ & $20,6 \pm 1,8$ aA & $(43 \%)$ \\
\hline & Total (g) & $45,7 \pm 2,5 \mathrm{a}$ & - & $129,2 \pm 9,0 \mathrm{bB}$ & - & $47,6 \pm 3,3 \mathrm{aA}$ & - \\
\hline & Relación BA/BR & $2,3 \pm 0,1 \mathrm{a}$ & - & $2,0 \pm 0,4 \mathrm{abA}$ & - & $1,4 \pm 0,3 \mathrm{bA}$ & - \\
\hline \multirow{5}{*}{ Peumo } & Hojas (g) & $20,8 \pm 2,6$ a & $(25 \%)$ & $35,4 \pm 5,4$ bB & $(25 \%)$ & $12,7 \pm 1,0 \mathrm{aA}$ & $(15 \%)$ \\
\hline & Ramas (g) & $26,0 \pm 2,4 \mathrm{a}$ & $(32 \%)$ & $47,8 \pm 5,5 \mathrm{bA}$ & $(33 \%)$ & $32,5 \pm 1,1 \mathrm{aA}$ & $(39 \%)$ \\
\hline & Raíz (g) & $35,3 \pm 1,9$ a & $(43 \%)$ & $60,7 \pm 14,4 \mathrm{aA}$ & $(42 \%)$ & $37,9 \pm 6,2 \mathrm{aA}$ & $(46 \%)$ \\
\hline & Total (g) & $82,1 \pm 6,2$ a & - & $143,9 \pm 25,3 \mathrm{bA}$ & - & $83,1 \pm 5,3$ aA & - \\
\hline & Relación BA/BR & $1,3 \pm 0,1 \mathrm{a}$ & - & $1,4 \pm 0,1 \mathrm{aA}$ & - & $1,3 \pm 0,2 \mathrm{aA}$ & - \\
\hline
\end{tabular}

*BA: biomasa aérea, BR: biomasa radical.

potenciales hídricos más bajos, lo cual es coincidente con la escasa variación observada entre los tratamientos en el contenido hídrico relativo a pre-alba durante el periodo de mantención de la restricción hídrica (cuadro 1). El ajuste del potencial osmótico a través de la acumulación activa de solutos ha sido ampliamente reconocido como una respuesta adaptativa a la menor disponibilidad de agua en el suelo. Poole y Miller (1978) mencionan que en condiciones naturales el peumo mantiene bajos potenciales osmóticos para generar un gradiente de potencial hídrico que le permitiría obtener el recurso hídrico disponible rápidamente.

Por otra parte, los valores de módulo de elasticidad observados en el periodo de mantención de la restricción hídrica, indican que tanto quillay como peumo no poseen capacidad de ajuste elástico como mecanismo de mantenimiento de la turgencia celular bajo condiciones de estrés hídrico, ya que sus valores fueron altos y no hubo diferencias entre tratamientos. Sin embargo, una situación diferente ocurrió en el periodo de rehidratación, en el que se detectaron diferencias entre tratamientos y entre especie, pues en quillay el módulo de elasticidad del tratamiento testigo fue inferior que el de restricción hídrica mientras que en peumo ocurrió lo contrario. En quillay, ello podría explicarse por el permanente crecimiento en longitud (figura 2C) que experimentaron las plantas del testigo durante este periodo debido al riego frecuente que recibieron, con la consecuente formación de nuevos brotes cuyas paredes celulares son más delgadas y elásticas que en células maduras. Un comportamiento similar en $\varepsilon$ observaron Salleo y Lo Gullo (1990) en algunas especies del género Quercus de clima mediterráneo, con un aumento progresivo en $\varepsilon$ a medida que aumenta el estado de desarrollo de las hojas. En cambio, en peumo la disminución de $\varepsilon$ observada en el tratamiento de restricción hídrica durante el periodo de rehidratación, es coincidente con lo observado en otras especies cuya respuesta en crecimiento sucede preferentemente después del estrés (Otieno et al. 2005). Los cambios requeridos a nivel de paredes celulares para llevar a cabo el ajuste elástico, que son mencionados por Zwiazek (1991), podrían explicar una respuesta de más largo plazo en el módulo de elasticidad. Si bien el ajuste osmótico y el ajuste elástico no se desarrollan en conjunto, o tienden a asumir uno mayor relevancia sobre el otro (Fan et al. 1994), ambos mecanismos requieren de posteriores estudios que aclaren la participación de estos en la regulación de la turgencia en peumo.

La disminución del contenido hídrico del sustrato, y la consiguiente disminución del potencial hídrico foliar, afecta la fisiología de quillay y peumo, provocaron una reducción en la fotosíntesis neta, la cual está determinada en principio por el cierre estomático. Este comportamiento es coincidente con lo observado en otras especies mediterráneas (Gulías et al. 2002, Peña-Rojas et al. 2004). En ambas especies se observó una relación directa entre fotosíntesis neta y conductancia estomática, lo cual coincidiría con lo mencionado por Cornic (2002) en el sentido de que la conductancia estomática sería el principal determinante en la disminución de la fotosíntesis neta. Sin embargo, el mayor valor de la concentración interna de $\mathrm{CO}_{2}$ observado en las plantas del tratamiento de restricción hídrica en peumo (figura $1 \mathrm{C}$ ), es un indicador de una limitación no estomática de la fotosíntesis. Esta limitación ha sido observada en otras especies mediterráneas como Quercus ilex L. y Pistacia lentiscus L. (Gulías et al. 2002, Peña-Rojas et al. 2004), y aunque es objeto de discusión esto podría es- 
tar determinado por una lenta regeneración de la ribulosa bifosfato (RuBP), lenta generación del adenosín trifosfato (ATP), por un mal funcionamiento de la rubisco, o por una menor conductancia del mesófilo (Lawlor 2002).

Las menores tasas fotosintéticas encontradas en peumo que en quillay, tanto en condiciones de riego como de estrés, parecen indicar que el balance de carbono es más favorable para esta última. Esta respuesta probablemente esté relacionada con la mayor pérdida de biomasa foliar que ocurrió en quillay, que se redujo en un $55 \%$ durante el periodo de estrés lo que pudo haber favorecido un mejor estatus hídrico de las hojas restantes y con ello una mayor fotosíntesis durante la restricción hídrica. Al respecto, Pinkard et al. (2007) mencionan que la abscisión de parte de las hojas trae como consecuencia un incremento en la tasa de fotosíntesis neta en las hojas restantes, mientras que Martínez y Armesto (1983) mencionan que los mayores potenciales hídricos y valores superiores de fotosíntesis bruta encontrados en quillay y litre, podrían estar relacionados con la mayor capacidad de estas especies para adquirir agua de los niveles más profundos del suelo, lo cual sería consecuencia de la gran capacidad de penetración de las raíces (Giliberto y Estay 1978). Esta capacidad de exploración parece tener relación con la disminución observada en la relación biomasa aérea/biomasa radical en quillay (cuadro 3), lo cual permitiría a la especie mantener una condición hídrica adecuada, permitiendo el funcionamiento de la fotosíntesis, aunque a una tasa reducida. En plantas bajo condiciones de déficit hídrico se ha documentado este aspecto, donde mayoritariamente se ha observado una disminución en la relación biomasa aérea/biomasa radical, ocasionada por un mayor crecimiento del sistema radical (McMillin y Wagner 1995). Mientras que la relación biomasa aérea/biomasa radical de peumo no fue afectada por la restricción hídrica, mientras que fotosíntesis neta es prácticamente cero en el tratamiento de restricción hídrica. Esta respuesta es semejante a la que presentan Acacia xanthophloea Benth. y varias especies mediterráneas del género Quercus bajo condiciones hídricas similares (Peña-Rojas et al. 2004, Otieno et al. 2005), e indicaría el uso de una estrategia de disminución de la pérdida de agua mediante el ajuste osmótico por sobre la inversión en recursos para la búsqueda de una fuente de agua. Esto es coherente con lo mencionado por Giliberto y Estay (1978), en el sentido de que peumo presenta un sistema radical superficial ( 0,5 a $1 \mathrm{~m}$ de profundidad), pero lateralmente extenso ( 3 - $4 \mathrm{~m}$ de radio), lo que le impide acceder a fuentes de agua más profundas.

\section{CONCLUSIONES}

Bajo condiciones de riego restringido, quillay no presenta un claro mecanismos de ajuste al déficit hídrico como ajuste osmótico y ajuste elástico, sino que al enfrentarse al periodo de estrés mas intenso, disminuye su biomasa foliar para equilibrar las pérdidas por transpiración, con la eficiencia del sistema radicular de suministrar agua a las hojas. Peumo en tanto, presenta una tendencia hacia el ajuste osmótico para mantener altos valores de contenido hídrico relativo y turgencia celular. Mayores estudios se requieren para verificar la capacidad de ajuste elástico en esta última especie.

Tanto quillay como peumo muestran respuestas negativas en los parámetros de intercambio gaseoso al encontrase bajo una condición de déficit hídrico. La fotosíntesis neta, conductancia estomática y transpiración, experimentan una drástica reducción en sus valores en las plantas bajo riego restringido respecto a las plantas del tratamiento testigo. En consecuencia, las plantas con riego restringido presentan un crecimiento y acumulación de biomasa sustantivamente menor al que presentan las plantas con riego constante.

Bajo condiciones de déficit hídrico, quillay disminuye significativamente la relación biomasa aérea/biomasa radical, debido a la defoliación que sufre y al incremento de la biomasa de raíces, mientras que en peumo la relación permanece invariable.

\section{AGRADECIMIENTOS}

Se agradece el financiamiento entregado por el Programa Domeyko-Biodiversidad de la Universidad de Chile y al Programa "Bosques Mediterráneos" de la Facultad de Ciencias Forestales y Conservación de la Naturaleza de la Universidad de Chile.

\section{REFERENCIAS}

Cabrera H. 2002. Respuestas ecofisiológicas de plantas en ecosistemas de zonas con clima mediterráneo y ambientes de altamontaña. Revista Chilena de Historia Natural 75: 625-637.

Chaves M, J Pereira, J Maroco, M Rodrigues, C Ricardo, M Osório, I Carvalho, T Faria, C Pinheiro. 2002. How plants cope with water stress in the field. Photosynthesis and growth. Annals of Botany 89: 907-916.

Cornic G. 2002. Drought stress inhibits photosynthesis by decreasing stomatal aperture - not by affecting ATP synthesis. Trends in Plant Science 5: 187-188.

Dickson R, P Tomlinson. 1996. Oak growth, development and carbon metabolism in response to water stress. Annals of Forest Science 53: 181-196.

Fan S, T Blake, E Blumwald. 1994. The relative contribution of elastic and osmotic adjustments to turgor maintenance of woody species. Physiologia Plantarum 90: 408-413.

Gallé A, P Haldimann, U Feller. 2007. Photosynthetic performance and water relations in young pubescent oak (Quercus pubescens) trees during drought stress and recovery. New Phytologist 174: 799-810.

Gebrekirstos A, D Teketay, M Fetene, R Mitlöhner. 2006. Adaptation of five co-occurring tree and shrub species to water stress and its implication in restoration of degraded lands. Forest Ecology and Management 229: 259-267.

Giliberto J, H Estay. 1978. Seasonal water stress in some chilean matorral shrubs. Botanical Gazzete 139: 236-240. 
Gulías J, J Flexas, A Abadía, H Madrano. 2002. Photosynthetic responses to water deficit in six mediterranean sclerophyll species: possible factors explaining the declining distribution of Rhamnus ludovici-salvatoris, an endemic Balearic species. Tree Physiology 22: 687-697.

Lawlor D. 2002. Limitations to photosynthesis in water-stressed leaves: stomata vs. metabolism and the role of ATP. Annals of Botany 89: 871-885.

Lawlor D, G Cornic. 2002. Photosynthetic carbon assimilation and associated metabolism in relation to water deficits in higher plants. Plant, Cell \& Environment 25: 275-294.

Lenz T, I Wright, M Westoby. 2006. Interrelations among pressure-volume curve traits across species and water availability gradients. Physiologia Plantarum 127: 423-433.

Martínez J, J Armesto. 1983. Ecophysiological plasticity and habitat distribution in three evergreen sclerophyllous shrubs of the chilean matorral. Oecologia Plantarum 4: 211-219.

McMillin J, M Wagner. 1995. Effects of water stress on biomass partitioning of Ponderosa Pine seedlings during primary root growth and shoot growth periods. Forest Science 41: 594-610.

Munné-Bosch S, L Alegre. 2004. Die and let live: leaf senescence contributes to plant survival under drought stress. Functional Plant Biology 31: 203-216.

Otieno D, M Schmidt, S Adiku, J Tenhunen. 2005. Physiological and morphological responses to water stress in two Acacia species from contrasting habitats. Tree Physiology 25: 361-371.

Peña-Rojas K, X Aranda, I Fleck. 2004. Stomatal limitation to
$\mathrm{CO}_{2}$ assimilation and down-regulation of photosynthesis in Quercus ilex resprouts in response to slowly imposed drought. Tree Physiology 24: 813-822.

Pinkard E, M Battaglia, C Mohammed. 2007. Defoliation and nitrogen effects on photosynthesis and growth of Eucalyptus globulus. Tree Physiology 27: 1053-1063.

Poole D, P Miller. 1978. Water related characteristics of some evergreen sclerophyll shrubs in central Chile. Oecologia Plantarum 13: 289-299.

Raftoyannis Y, K Radoglou. 2002. Physiological responses of beech and sessile oak in a natural mixed stand during a dry summer. Annals of Botany 89: 723-730.

Rodríguez R, S Matthei, M Quezada. 1983. Flora arbórea de Chile. Concepción, Chile. Editorial de la Universidad de Concepción. 408 p.

Salleo S, M Lo Gullo. 1990. Sclerophylly and plant water relations in three mediterranean Quercus species. Annals of Botany 65: 259-270.

Vilagrosa A, J Cortina, E Rubio, R Trubat, E Chirino, E GilPelegrín, V Vallejo. 2005. El papel de la ecofisiología en la restauración forestal de ecosistemas mediterráneos. Investigación Agraria: Sistemas y Recursos Forestales 14: 446-461.

Woodruff D, B Bond, F Meinzer. 2004. Does turgor limit growth in tall trees?. Plant, Cell \& Environment 27: 229-236.

Zwiazek J. 1991. Cell wall changes in white spruce (Picea glauca) needles subjected to repeated drought stress. Physiologia Plantarum 82: 513-518.

Recibido: 10.09 .10

Aceptado: 26.05.11 
